\title{
TWO RELATED ESTIMATION PROBLEMS
}

\author{
W. BRISLEY
}

(Received 11 July 1977)

(Revised 19 October 1977)

\begin{abstract}
Two problems involving the "best" solution $X$ for a matrix equation $V X V^{*}=M$ are discussed, together with methods for their solution, and a generalization of one of the methods beyond matrix equations.
\end{abstract}

\section{The two problems}

Suppose $V$ and $M$ to be given $k \times n$ and $k \times k$ matrices respectively, and consider the equation

$$
V X V^{*}=M
$$

(where * indicates complex conjugate transpose-in general, the matrices will be taken over $C$ ).

In typical cases, this equation arises with $k<n$, and there is no diagonal solution $X$, the matrix $M$ being a "distortion" of a result derived from a supposed diagonal matrix, the "distortion" being due to noise or some other factor. For a particular case in signal processing, see, for example, [1].

Two problems arise:

Problem (a). Select, from the set of all solutions $X$ of (1), that particular matrix $X$ for which the quantity

is least.

$$
\sum_{\substack{i \neq j \\ i=1 \ldots n \\ j=1 \ldots n}}\left|x_{i j}\right|^{2}
$$

(If such a solution exists, we will term it the "tightest" solution of (1): one may reasonably describe it as being the solution which is "closest to being diagonal". 
In typical cases, the matrix $V$ is usually arranged from experimental data, and is of rank $k$; in this circumstance (1) has solutions and so the problem makes sense. For a substantial discussion of further circumstances under which solutions may exist, see Rao [2].)

Problem (b). Select, from the set of all diagonal $n \times n$ matrices, $D$, that particular one $D$ such that the quantity $\left|V D V^{*}-M\right|$ is least, where $|A|$ denotes

$$
\sum_{\substack{i=1 \ldots k \\ i=j \ldots k}}\left|a_{i j}\right|^{2}
$$

(One may reasonably describe it as "the diagonal which is closest to giving $M$ ". Even if (1) has no solutions, this problem still makes sense, and then may be the most appropriate one for some signal processing estimation tasks (d'Assumpcao [1]).)

In each case, it is clear that a necessary condition for the problem to be well-posed is that the equation

$$
V K V^{*}=0
$$

admits no diagonal non-zero solutions-for, if " $K=D^{\prime}$ " was such a solution, then whenever " $X=S$ " solved (a), then so would " $X=S+D$ '", and, whenever " $D=B$ " solved (b), then so would " $D=B+D$ ". Consequently, in assuming that the problems are well-posed, we assume that

$$
\text { If } V K V^{*}=0_{k \times k} \text { and } K \text { is diagonal, then } K=0_{n \times n} \text {. }
$$

(We shall return to some analysis of the conditions under which (3) holds, in Section 4.)

\section{Solution of Problem (a)}

We assume that (1) does not lack solutions, and adopt the point of view that (1), (2) and (3) are referring to the linear map $\phi$ from the space of $n \times n$ matrices to the space of $k \times k$ matrices, where

$$
\phi(A)=V A V^{*} .
$$

Consequently, the solution set of (2) is the kernel of $\phi$, statement (3) is a property of that kernel, and we are considering a search amongst all matrices of the form

$$
X=P+K,
$$

where $P$ is a particular matrix such that $\phi(P)=M$, and $K$ is such that $\phi(K)=0$.

Let $G$ be any "generalized inverse" for $V$; that is, let $G$ be one of the many $n \times k$ matrices such that

$$
V G V=V
$$


(and hence also

$$
\left.V^{*} G^{*} V^{*}=V^{*}\right) \text {. }
$$

The matrix $G$ has only to satisfy (4) for the following, so any of the "generalized inverses" defined in the literature will suffice (see, for example, Rao [3], pp. 24-26 and also Noble and Daniel [4]). The equation (4) implies that

$$
V \mathbf{k}=\mathbf{0} \text { if and only if } \mathbf{k}=(I-G V) \mathbf{r}
$$

for some arbitrary vector $\mathbf{r}$ and

$$
\begin{aligned}
& \text { if } V \mathbf{x}=\mathbf{b} \text { has solutions, then } \\
& \mathbf{x}=G \mathbf{b} \text { is one of them. }
\end{aligned}
$$

It follows from ( $\left.4^{\prime \prime}\right)$ that $G M G^{*}$ is a particular solution for (1). Letting $K$ denote any solution of (2) (that is, $K \in \operatorname{ker} \phi)$, we have from $\left(4^{\prime \prime}\right)$ that $K V^{*}=(I-G V) A$ for some arbitrary $n \times k$ matrix $A$, and hence (using (4") again) that

$$
K=(I-G V) A G^{*}+B\left(I-V^{*} G^{*}\right),
$$

where $A$ and $B$ can be any $n \times k$ and $n \times n$ matrices. Adopting $P=G M G^{*}$, we need only select $K$, which we do using a Fourier technique, as follows.

Suppose $V$ has rank $r$. Then the subspace of $C^{n}\{\mathbf{k} \mid V \mathbf{k}=0\}$ has a basis $\left\{\mathbf{k}_{1}, \mathbf{k}_{2}, \ldots, \mathbf{k}_{v}\right\}$ with $\nu=n-r$ (note that a suitable basis will have already appeared in the calculation of $G$ ). Let $C_{i j}$ denote the $n \times n$ matrix which has $\mathbf{k}_{j}$ entered as column $i$, and zero for all other entries. Then clearly any matrix of the form

$$
\sum_{\substack{i=1 \ldots n \\ j=1 \ldots v}} \alpha_{i j} C_{i j}+\sum_{\substack{i=1 \ldots n \\ j=1 \ldots v}} \beta_{i j} C_{i j}^{*}
$$

will be a solution of (2). Since (5) displays each such $K$ as a sum

$$
K=R+S \text { with } V R=0 \text { and } S V^{*}=0
$$

it follows that $\operatorname{ker} \phi$ is spanned by the set $\mathscr{K} \cup \mathscr{K}^{*}$, where

$$
\mathscr{K}=\left\{C_{i j}\right\}_{\substack{i=1 \ldots n \\ j=1 \ldots v}}, \mathscr{K}^{*}=\left\{C_{\substack{i j \\ j=1 \ldots n \\ j=1 \ldots v}}^{*}\right.
$$

On the space of all $n \times n$ matrices, set up the inner product $\langle;\rangle$ by defining that

$$
\langle A ; B\rangle=\sum_{i \neq j} a_{i j} \overline{b_{i j}} \text {. }
$$

It is clear that this is an inner product, that it is not positive definite, but also that:

when restricted to $\operatorname{ker} \phi$, under assumption (3), $\langle;\rangle$ is a positive definite inner product on $\operatorname{ker} \phi$. 
One can then define $\|X\|$ to mean $\sqrt{ }(\langle X ; X\rangle)$ and note that $\|X\|$ is 0 if and only if $X$ is diagonal.

Although $\mathscr{K} \cup \mathscr{K}^{*}$ is not necessarily a linearly independent set, it will yield (by, for example, a modified Gram-Schmidt process) an orthonormal basis for $\operatorname{ker} \phi$, say

$$
\left\{K_{1}, K_{2}, \ldots, K_{m}\right\} \text { with }\left\langle K_{i}, K_{j}\right\rangle=\delta_{i j}, \quad m \leqslant 2 n \nu .
$$

Then any solution of (1) reads

$$
\text { " } X=S \text { where } S=P+\sum_{i=1}^{m} \alpha_{i} K_{i} "
$$

and Problem (a) is reduced to selecting the $\alpha_{i}$ to minimize $\|S\|$. Since $P$ has been adopted already, and hence the numbers $\langle P ; P\rangle$ and $f_{i}=\left\langle P ; K_{i}\right\rangle$ are fixed, we calculate that

$$
\begin{aligned}
\langle S ; S\rangle & =\langle P ; P\rangle+\sum_{1}^{m} \alpha_{i} \bar{f}_{i}+\sum_{1}^{m} \bar{\alpha}_{i} f_{i}+\sum_{1}^{m} \alpha_{i} \bar{\alpha}_{i}, \\
& =\langle P ; P\rangle+\sum_{i=1}^{m}\left(\alpha_{i}+f_{i}\right)\left(\bar{\alpha}_{i}+\bar{f}_{i}\right)-\sum_{i=1}^{m} f_{i} \bar{f}_{i}
\end{aligned}
$$

Thus, a minimum for $\|S\|$ is achieved if and only if each $\alpha_{i}$ is $-f_{i}$, and the solution to problem (a) appears as

$$
\text { “ } X=S \quad \text { with } \quad S=P-\sum_{i=1}^{m}\left\langle P ; K_{i}\right\rangle K_{i} " .
$$

(That the result is independent of the particular choice of $P$ is clear, since if $S_{j}=P_{j}-\sum_{i=1}^{m}\left\langle P_{j} ; K_{i}\right\rangle K_{i}(j=1,2)$ then, since $P_{1}-P_{2} \in \operatorname{ker} \phi$, and hence

$$
P_{1}-P_{2}=\sum_{i=1}^{m}\left\langle\left(P_{1}-P_{2}\right) ; K_{i}\right\rangle K_{i}
$$

we have $S_{1}-S_{2}=0$. It is equally "standard" that the result is independent of the particular orthonormal basis chosen for $\operatorname{ker} \phi$. Thus, it does make sense to speak of the tightest solution $X=S$ given by (8).)

Some further remarks are of interest:

(i) If $M$ of (1) is Hermitian, then the "tightest" solution is also Hermitian.

This can be seen as follows: from (6), we have

$$
S^{*}=P^{*}-\sum_{i=1}^{m}\left\langle\overline{P ; K_{i}}\right\rangle K_{i}^{*}
$$


But since $\langle\overline{A ; B}\rangle=\left\langle A^{*} ; B^{*}\right\rangle$, the set $\left\{K_{1}^{*}, \ldots, K_{n}^{*}\right\}$ is also an orthonormal basis for $\operatorname{ker} \phi$, and since $P^{*}$ is also a particular solution of (1) when $M$ is Hermitian, then $S^{*}$ is merely the "tightest solution" using $P^{*}$ and $\left\{K_{1}^{*}, \ldots, K_{n}^{*}\right\}$. Hence $S=S^{*}$.

(ii) It can be arranged that each $K_{i}$ is Hermitian, and hence, if $M$ is Hermitian, that each $\left\langle P ; K_{i}\right\rangle$ be real.

We note that the Gram-Schmidt process preserves "Hermitian", since it only involves calculations süch as $A\langle\langle A ; A\rangle$ and $A-\langle A ; B\rangle$, and $\langle A ; B\rangle$ is real if both $A$ and $B$ are Hermitian. If then, we use, instead of $\mathscr{K} \cup \mathscr{K}^{*}$, the spanning set

$$
\left\{\left(C_{i j}+C_{i j}^{*}\right)\right\}_{\substack{i=1 \ldots n \\ j=1 \ldots v}} \cup\left\{i\left(C_{l s}-C_{i j}^{*}\right)\right\}_{\substack{l=1 \ldots n \\ s=1 \ldots v}}
$$

(which consists entirely of Hermitian matrices) and apply the Gram-Schmidt process, the resulting $\left\{K_{1}, \ldots, K_{n}\right\}$ will consist entirely of Hermitians. If, further, $M=M^{*}$, and we use $P=G M G^{*}$, then $P^{*}=P$, and hence

$$
\left\langle\overline{P ; K_{i}}\right\rangle=\left\langle P^{*} ; K_{i}^{*}\right\rangle=\left\langle P ; K_{i}\right\rangle .
$$

(iii) In the special case that $V, M$ are real, and $M$ is symmetric, we need only consider real symmetric matrices, in which case the dimension of $\operatorname{ker} \phi$ is no greater than $n \nu$.

For, by remark (ii), we have collapse from "Hermitian" to "symmetric" whereever appropriate. If $K$ is in $\operatorname{ker} \phi$, it can be written as some linear combination

$$
K=\sum_{\substack{i=1 \ldots n \\ j=1 \ldots v}} \alpha_{i j} C_{i j}+\sum_{\substack{i=1 \ldots . . n \\ j=1 \ldots v}} \beta_{i j} C_{i j}^{\mathrm{T}} \text {. }
$$

But also $K=K^{\mathrm{T}}=\sum \beta_{i j} C_{i j}+\sum \alpha_{i j} C_{i j}^{\mathrm{T}}$ whence $K=\sum \gamma_{i j}\left(C_{i j}+C_{i j}^{\mathrm{T}}\right)$ where $\gamma_{i j}=\frac{1}{2}\left(\alpha_{i j}+\beta_{i j}\right)$. Hence, we may take $\left\{\left(C_{i j}+C_{i j}^{T}\right)\right\}_{i=1 \ldots n}$ as a spanning set. That dimension $n \nu$ can be attained is demonstrated by the following example.

giving

$$
V=\left[\begin{array}{cccc}
1 & 0 & 0 & -1 \\
0 & 1 & 0 & -1 \\
0 & 0 & 1 & -1
\end{array}\right] ; \quad \nu=1, \quad \mathbf{k}_{1}=\left[\begin{array}{l}
1 \\
1 \\
1 \\
1
\end{array}\right]
$$

$$
K_{1}=\frac{1}{\sqrt{6}}\left[\begin{array}{cccc}
2 & 1 & 1 & 1 \\
1 & & & \\
1 & & 0 & \\
1 & &
\end{array}\right], \quad K_{2}=\frac{1}{4 \sqrt{3}}\left[\begin{array}{cccc}
-2 & 2 & -1 & -1 \\
2 & 6 & 3 & 3 \\
-1 & 3 & 0 & 0 \\
-1 & 3 & 0 & 0
\end{array}\right],
$$




$$
K_{3}=\frac{1}{4 \sqrt{5}}\left[\begin{array}{cccc}
-2 & -2 & 3 & -1 \\
-2 & -2 & 3 & -1 \\
3 & 3 & 8 & 4 \\
-1 & -1 & 4 & 0
\end{array}\right], \quad K_{4}=\frac{1}{\sqrt{30}}\left[\begin{array}{cccc}
-1 & -1 & -1 & 2 \\
-1 & -1 & -1 & 2 \\
-1 & -1 & -1 & 2 \\
2 & 2 & 2 & 5
\end{array}\right]
$$

giving, when

$$
M=\left[\begin{array}{lll}
2 & 1 & 0 \\
1 & 2 & 1 \\
0 & 1 & 2
\end{array}\right],
$$

the "tightest solution" for Problem (a) as

$$
S=\frac{1}{8}\left[\begin{array}{cccc}
10 & 1 & -2 & 1 \\
1 & 4 & 1 & -2 \\
-2 & 1 & 10 & 1 \\
1 & -2 & 1 & 4
\end{array}\right]
$$

\section{Solution of Problem (b)}

Here, we take the view that $V$ affords a linear map $\psi$ from the space of all diagonal $n \times n$ matrices into the space of all $k \times k$ matrices, by

$$
\psi(D)=V D V^{*} \text {. }
$$

Condition (3) assures us that $\psi$ is of rank $n$, and Problem (b) is solved by finding that matrix $Q$ in Image $\psi$ which is "closest" to $M$, and then setting $D=\psi^{-1}(Q)$. In this case, the relevant inner product on the space of $k \times k$ matrices is

$$
\langle\langle A ; B\rangle\rangle \text { defined as } \sum_{\substack{i=1 . \ldots k \\ j=1 \ldots k}} a_{i j} \bar{b}_{i j}
$$

(so that the corresponding norm $|\cdot|$ is as in Problem (b)). Calculating

$$
\left\{\psi\left(D_{1}\right), \psi\left(D_{2}\right), \ldots, \psi\left(D_{n}\right)\right\}
$$

for an appropriate linearly independent set of diagonals gives a basis for Image $\psi$, hence an orthonormal basis for Image $\psi$, say $\left\{B_{i}\right\}_{i=1, \ldots, n}$, and then $Q$ is determined uniquely as

$$
Q=\sum_{i=1}^{n}\left\langle\left\langle M ; B_{i}\right\rangle\right\rangle B_{i} .
$$

(By arguments similar to previous ones, we may take the $B_{i}$ to be Hermitian, and so if $M$ is Hermitian, all the $\left\langle\left\langle M ; B_{i}\right\rangle\right\rangle$ are real, and $Q$ is Hermitian.) 
The equation

can be rewritten as

$$
\psi(D)=Q
$$

$$
N \mathbf{d}=\mathbf{q},
$$

where $\mathrm{d}$ is the diagonal of $D, \mathbf{q}$ is a suitable "unwrapping" of $Q$ and $N$ is $k^{2} \times n$. Since $N$ is of rank $n$ by (3), we have $\mathbf{d}=Z \mathbf{q}$ whenever $Z$ is a generalized inverse for $N$. Equation (10) and condition (3) are discussed below.

As a matter of comparison with Problem (a), the process applied to the same $V, M$ in the illustration given yields

$$
\left\{B_{1}=\left[\begin{array}{lll}
1 & 0 & 0 \\
0 & 0 & 0 \\
0 & 0 & 0
\end{array}\right], \quad B_{2}=\left[\begin{array}{lll}
0 & 0 & 0 \\
0 & 1 & 0 \\
0 & 0 & 0
\end{array}\right], \quad B_{3}=\left[\begin{array}{lll}
0 & 0 & 0 \\
0 & 0 & 0 \\
0 & 0 & 1
\end{array}\right], \quad B_{4}=\frac{1}{\sqrt{6}}\left[\begin{array}{lll}
0 & 1 & 1 \\
1 & 0 & 1 \\
1 & 1 & 0
\end{array}\right]\right\}
$$

and hence

$$
D=\frac{1}{3}\left[\begin{array}{llll}
4 & & & 0 \\
& 4 & & \\
& & 4 & \\
0 & & & 2
\end{array}\right]
$$

as a solution for Problem (b).

\section{The "well-posing" of the problems}

Condition (3) is clearly necessary for the problems to be well-posed. Since, with it, they each have unique solutions, it is also sufficient for them to be well-posed, as long as, in Problem (a), we assume the existence of at least one solution $X$ to equation (1). The equations (10) and (10') (and hence condition (3)) can be explicitly written in numerous ways: one such is to "unwrap" $Q$ as the column whose entries read in order

$$
q_{11}, q_{12}, \ldots, q_{1 k}, q_{21}, \ldots, q_{2 k}, q_{31}, \ldots, q_{k 1}, \ldots, q_{k k} \text {. }
$$

In this case (the diagonal $d$ reading $d_{11}, d_{22}, \ldots, d_{n n}$ ) one has $N$ consisting of $k^{2}$ rows, the $i$ th block of $k$ rows reading:

$$
\begin{aligned}
& v_{i 1} \bar{v}_{11}, v_{i 2} v_{12}, \ldots, v_{i n} \bar{v}_{1 n}, \\
& v_{i 1} \bar{v}_{21}, v_{i 2} \bar{v}_{22}, \ldots, v_{i n} \bar{v}_{2 n}, \\
& \vdots \\
& v_{i 1} \bar{v}_{k 1}, v_{i 2} \bar{v}_{k 2}, \ldots, v_{i n} \bar{v}_{k n} .
\end{aligned}
$$


For condition (3), that is, for $N$ to be rank $n$, we clearly need at least, that $n \leqslant k^{2}$. (If $N$ is of rank $n$, with $n=k^{2}$, then equation (1) has a unique diagonal solution, and both problems become, trivially, "solve $N \mathbf{d}=\mathbf{m}$ where $\mathbf{m}$ is $M$ unwrapped".) If $M$ is Hermitian, then the $Q$ of $(10)$ is Hermitian, and so $\psi(D)=Q$ implies that $D$ must be real; since the rows of $N$ occur in complex conjugate pairs, this leaves just $\frac{1}{2} k(k+1)$ equations to solve, $k$ of them being real : although this does not alter the bound on the necessary rank of $N$, it does provide a smaller matrix than $N$ for the computation of the appropriate generalized inverse.

Two special cases are worthy of mention. In at least one type of application, each entry in $V$ is of modulus 1 , so that $N$ has at least $k$ rows with each entry 1. In this case, then, one has " $k^{2}-k+1 \geqslant n$ " as a necessary condition for each of the problems to be well-posed, and $M$ not having equal diagonal entries is then a sufficient condition for there to be no diagonal solution to equation (1).

In applications where all matrices are real, there will be at most $\frac{1}{2} k(k+1)$ distinct rows in $N$, so a necessary condition for the problems to be well-posed is that $n \leqslant \frac{1}{2} k(k+1)$. If, further, $M$ is symmetric, $n$ being $\frac{1}{2} k(k+1)$ will render both problems trivial, in that (1) will then have a unique diagonal solution.

Further discussion of the relations between the rank of $N$ and properties of $V$ can be found in [2].

\section{Generalization of Problem (a) and its solution}

The Problem (a) is a special case of the following: given the linear map $\phi: U \rightarrow V$, with $S$ a subspace of the vector space $U$, and $\mathbf{g}$ in $V$, find that solution $\mathbf{x}$ of $\phi(\mathbf{x})=\mathbf{g}$ such that $\mathbf{x}$ is "closest possible" to being a member of $S$.

If we take it that $\langle;\rangle$ is a positive definite inner product on $U$, so that $U$ splits as $S \oplus S^{\perp}$ (each $\mathbf{u}$ in $U$ being written $\mathbf{u}=s(\mathbf{u})+r(\mathbf{u})$ uniquely, with $s(\mathbf{u}) \in S$, $r(u) \in S^{\perp}$ ), the obvious measure is to define

$$
\langle\langle\mathbf{u} ; \mathbf{v}\rangle \overline{\rangle}=\langle r(\mathbf{u}) ; r(\mathbf{v})\rangle
$$

so that $\langle\langle;\rangle\rangle$ is a positive definite inner product on the factor space $U / S$ and, also, on $K / K \cap S$ where $K$ is the kernel of $\phi$. Taking $\left\{k_{1}, k_{2}, \ldots\right\}$ (modulo $K \cap S$ ) as an orthonormal basis for $K / K \cap S$, with respect to $\langle\langle;\rangle\rangle$, it follows (as in the particular case of Section 2) that of all possible solutions of $\phi(x)=\mathbf{g}$, that solution with $\langle\langle\mathbf{x} ; \mathbf{x}\rangle\rangle$ least possible is

$$
\mathbf{x}=\mathbf{p}-\Sigma\left\langle\left\langle\mathbf{p} ; \mathbf{k}_{i}\right\rangle\right\rangle \mathbf{k}_{\boldsymbol{i}} \quad(\operatorname{modulo} K \cap S),
$$

where $\mathbf{p}$ is any particular solution to $\phi(\mathbf{p})=\mathbf{g}$. 
Perhaps the simplest examples (other than the more obvious signal processingparameter estimation problems) are in control problems; as two samples which are simple enough for scratch pad checking, we have:

(i) Find the input $y(t)$ with least R.M.S. over $0 \leqslant t \leqslant 1$, such that $y-y^{\prime}=t^{2}-2 t$. (Here $S$ is zero, $K$ is spanned by the single function $e^{l}$, and the required function is $y(t)=t^{2}-\left(2(e-2) /\left(e^{2}-1\right)\right) e^{t}$.)

(ii) Find the input $y(t)$, which is closest to being linear such that $y-y^{\prime}=t^{2}-2 t$ in the intervai $0 \leqslant t \leqslant 1$. (Here one takes $S$ as the subspace of all linear functions, with $\langle f ; g\rangle$ being $\int_{0}^{1} f g d t$, so that the required function is $y(t)=t^{2}-(1 / 3(3-e)) e^{t}$.)

The usefulness of the method, in this context, increases with the degree of the relevant differential equation, since it merely involves the projection of a new particular solution on to the fixed space $K / K \cap S$ each time a "new" target function is presented, rather than re-solving a "new" minimization problem. In practice, the parameters needed are immediately available by quadrature, whereas the corresponding $a b$ initio minimization problem involves not only quadratures but also the solution of a set of simultaneous equations which are often, because of their source, fairly badly conditioned.

\section{Acknowledgement}

The author wishes to thank the referee for his comments and suggestions.

\section{REFERENCES}

[1] H. A. d'Assumpcao, "Estimation of sound directionality", Australian Weapons Research Establishment WRE-TN-1369 (WR \& D).

[2] C. R. Rao, "Estimation of heteroscedastic variance", J. Amer. Statist. Ass. 65 (1970), 161-172.

[3] C. R. Rao, Linear statistical inference (John Wiley \& Sons Inc., New York 1965).

[4] B. Noble and J. W. Daniel, Applied linear algebra (Prentice Hall, New York 2nd ed., 1977).

Department of Mathematics

University of Newcastle

New South Wales 2308

Australia 